\title{
Effect of Inter-Repetition Rest vs. Traditional Resistance Training on the Upper Body Strength Rate of Force Development and Triceps Brachii Muscle Architecture
}

\author{
by \\ Nikolaos Zaras ${ }^{1,2}$, Angeliki-Nikoletta Stasinaki', Thomas Mpampoulis', \\ Polyxeni Spiliopoulou' ${ }^{2}$, Marios Hadjicharalambous ${ }^{1}$, Gerasimos Terzis ${ }^{2}$
}

The purpose of the study was to examine the effect of seven-week inter-repetition rest vs. traditional resistance training on upper body maximum strength, the rate of force development and triceps brachii muscle architecture. Sixteen male participants were equally assigned into the inter-repetition rest and the traditional group. In both groups, training included the bench press exercise performed with 4 sets of 6 maximum repetitions, two training sessions per week. Twenty-second inter-repetition rest was employed for the inter-repetition rest group only. Measurements before and after the training period included maximum strength in the bench press, the isometric upper body rate of force development and peak force and triceps brachii muscle architecture. Maximum strength increased significantly in both groups (inter-repetition rest group: $21.5 \pm 5.7 \%$ vs. traditional group: $13.5 \pm 7.2 \%, p<0.05$ ), however, the maximum strength percentage increase was greater in the inter-repetition rest group compared to the traditional group ( $p=$ 0.027). Upper body isometric peak force increased only after inter-repetition rest training $(10.7 \pm 10.3 \%, p=0.009)$. The rate of force development remained unchanged for both groups $(p>0.05)$, although percentage changes in time frames of 0-80 and 0-100 milliseconds were greater for the inter-repetition rest group compared to the traditional training group ( $p=0.024$ and $p=0.044$, respectively). Triceps brachii thickness increased similarly for both groups ( $p<$ 0.05). These results suggest that inter-repetition rest may induce greater increases in maximum strength and the rate of force development compared to traditional training during the initial weeks of resistance training.

Key words: resistance training, upper body power, bench press.

\section{Introduction}

Resistance exercise is a proven training intervention to increase muscle strength and mass, with various combinations of the acute training variables resulting in significant strength improvements. Usually, a number of repetitions are performed in a certain exercise with a load between $30-90 \%$ of maximal strength, followed by a rest period of 1-3 minutes (Golas et al., 2019; González-Badillo et al., 2014). However, it has been proposed that the introduction of a few second rest (e.g. 15-45 s) between single repetitions or after clusters of 2-3 repetitions in a single set, may result in faster adenosine triphosphate (ATP) resynthesis and lower concentration of muscle lactate, which allows the use of higher training intensity/volume and finally, greater longitudinal increases in muscle strength (Haff et al., 2008; Iglesias-Soler et al., 2012; Nickolson et al., 2016). Indeed, this resistance training strategy allows for a higher total training intensity and volume compared to traditional resistance training which may also result in greater increases in velocity and power production (Inglesias-Soler et al., 2014; Torrejón et al., 2019).

1 - Human Performance Laboratory, Department of Life and Health Sciences, University of Nicosia, Cyprus.

2 - Sports Performance Laboratory, School of Physical Education and Sport Science, National and Kapodistrian University of Athens, Greece. 
Inter-repetition rest and cluster resistance training have been successfully used to increase lower body strength (Nicholson et al., 2016; Oliver et al., 2013), however, whether inter-repetition rest training increases upper body muscle strength remains debated. Traditional resistance training increases elbow flexors' maximum strength by approximately $15.1 \%$ more than cluster training (Rooney et al., 1994). In another report, bench press strength (6-RM) was increased by $9.4 \%$ after traditional vs. $4.7 \%$ after cluster resistance training in a group of elite junior basketball and soccer athletes, following six weeks of training (Lawton et al., 2004). Additionally, five weeks of whole body resistance training including the bench press exercise with 16 sets of 2 repetitions with 1-min short intervals, induced similar increases in maximum upper body strength compared to traditional resistance training performed with 4 sets of 8 repetitions in male and female physical education students (Rial-Vázque et al., 2020). Likewise, Davies et al. (2019) found similar increases in bench press maximum strength following eight weeks of whole body traditional and inter-repetition rest set configurations in regularly resistance trained males and females. In contrast, Oliver et al. (2013) reported that cluster training induced a higher increase in bench press strength $(15.1 \%)$ compared to traditional resistance training $(9.1 \%)$, following a whole-body resistance training program in a group of active duty military resistance-trained men. Considering the above ambiguous results, it seems uncertain whether inter-repetition rest resistance training is more effective compared to traditional resistance training in increasing upper body strength. This may be due to the different training background of participants and/or concurrent resistance training of other body parts, which might have induced divergent neural adaptation. Neural adaptation may have interfered with the strength outcome in these studies because of their relative short duration which suggests strong neural involvement (Komi, 2004). Practicing a single resistance exercise for the upper body, e.g., the bench press, in participants of the same gender and similar training background might provide a clearer picture of the effect of inter-repetition rest resistance training on strength development.

The bench press is a multijoint exercise habitually used in resistance training to stimulate upper body muscle strength and mass and to test upper body strength (Miller, 2012). The bench press exercise activates the major and minor pectoralis muscles, medial and anterior deltoids and triceps brachii among other upper body muscles (Krzysztofik et al., 2021; Stastny et al., 2017). When pectoral muscles are fatigued during the bench press, the activation of triceps brachii is increased (Brennecke et al., 2009), implying that the triceps brachii may receive a strong hypertrophic stimulus with the continuing number of repetitions. Indeed, long-term traditional resistance training including the bench press exercise results in significant increases in triceps brachii muscle thickness (Mata et al., 2012; Ogasawara et al., 2012). Resistance training with inter-repetition rest may induce to some extent different muscle hypertrophy in the triceps brachii muscle because of the anticipated lower fatigue of the pectoral and triceps muscles due to the additional rest between repetitions during the bench press. However, the effect of bench press training with inter-repetition rest on triceps brachii muscle characteristics remains unknown.

The rate of force development (RFD) describes the ability of the neuromuscular system to produce high amounts of force within short time-frames (e.g. 0-250 ms) and is a valid indicator of performance in power demanding sports (Zaras et al., 2021). Traditional resistance training increases the lower body RFD as long as there is an intention of fast movement during the concentric phase (Blazevich et al., 2020). However, scarce data exist regarding the effect of resistance exercise on the upper body RFD. Fourteen weeks of bench press strength/power resistance training failed to increase the upper body RFD in moderately-trained participants (Hartmann et al., 2014). To our knowledge, the effect of resistance training with inter-repetition rest on the RFD, especially of the upper body, remains unexplored.

Therefore, the purpose of the present study was to investigate the effects of interrepetition rest versus traditional resistance training on upper body maximum strength, the RFD and triceps brachii muscle architecture. It was hypothesized that inter-repetition rest resistance training would induce greater increases in upper body maximum strength and the RFD, while both training programs would induce 
similar changes in triceps brachii muscle characteristics.

\section{Methods}

\section{Participants}

Sixteen male physical education students with resistance training experience of $3.6 \pm 2.1$ years and $102.3 \pm 21.3 \mathrm{~kg}$ personal best performance in the bench press participated in the study. Participants were assigned into two groups, the inter-repetition rest group (IRRG: $\mathrm{N}=$ 8, age: $23.6 \pm 6.8$ years, body mass: $75.7 \pm 8.4 \mathrm{~kg}$, body height: $1.80 \pm 0.07 \mathrm{~m}$ ) and the traditional group (TRG: $\mathrm{N}=8$, age: $21.7 \pm 2.7$ years, body mass: $79.5 \pm 12.8 \mathrm{~kg}$, body height: $1.79 \pm 0.08 \mathrm{~m}$ ), according to their initial 1-RM strength in the bench press (IRRG: $93.1 \pm 15.7 \mathrm{~kg}$ vs. TRG: $93.9 \pm$ $7.9 \mathrm{~kg}, \quad p=0.898$ ). Participants signed an institutionally approved informed consent form before entering the experimental procedures. Participants were in good health, they were not consuming nutritional supplements and they refrained from any systematic resistance training program for at least 6 months prior to the study entry. All procedures were in accordance with the 1975 Declaration of Helsinki, as revised in 2000, and were approved by the institutional ethics committee (project number 1024/8/11/2017).

\section{Design and Procedures}

Before and after the training period, participants visited the laboratory on different occasions, $48 \mathrm{~h}$ apart. During their first visit, measurement of 1 repetition maximum (RM) strength on the bench press and a familiarization session on upper body RFD measurement were performed. The second and the third visits included the measurement of the upper body RFD and isometric peak force (PF), as well as the evaluation of triceps brachii muscle architecture, respectively. All measurements were performed in the same order during the end of the training period.

Training: Training was performed for seven weeks, twice per week with at least 72 hours rest between each training session. The TRG performed 4 sets of 6 repetitions with approximately $85 \%$ of 1 -RM with a 3 min rest interval between sets. When participants of the TRG performed more than 6 repetitions during a training session, the load was increased to meet the 6 repetition criterion. As in the TRG, participants of the IRRG performed 4 sets of 6 repetitions with approximately $85 \%$ of $1-\mathrm{RM}$ using single repetitions with 3 min rest intervals between sets. Additionally, in the IRRG group, 20 $\mathrm{s}$ inter-repetition rest was applied (Hardee et al., 2012). Single repetitions were selected because of the previously reported positive effect on muscle strength (Nickolson et al., 2016). During this interrepetition rest period the barbell was locked back to the rack of the bench and the participant remained in the supine position with arms resting on the body-trunk. Five seconds before the end of the $20 \mathrm{~s}$ rest interval, participants placed their hands on the barbell and initiated the next repetition with a vocal signal of one researcher exactly at $20 \mathrm{~s}$. During all training sessions, two researchers assisted participants to lift the load from the bench rack to avoid unnecessary fatigue. Thirty minutes after the end of every training session, participants provided a score in a 1-10 scale of the rate of perceived exertion (RPE, Borg, 1982). When participants of the IRRG reported a score lower or equal to 7 , the load during the next training session was increased by $2.5 \%$ (relatively to the initial 1-RM) (Day et al., 2004; Sweet et al., 2004).

Bench press 1-RM strength: Bench press 1-RM strength testing was performed on a parallel bench. After a 10-min warm-up on a treadmill followed by static stretching and ten explosive push ups, participants performed 2 sets of 10 repetitions of the bench press exercise with $40 \mathrm{~kg}$ to warm-up (similarly to the warm-up routine during training). Then, 3 sets of 8,6 and 4 repetitions with approximately $50-60 \%, 70-75 \%$ and $80-85 \%$, respectively, of the predicted 1-RM were performed. Thereafter, 3-4 sets of 1 repetition were performed for determining maximum strength (1-RM) with 2-3 min rest intervals between sets. Two of the researchers were present for monitoring the technique of the exercise, assisting all participants during lifting and encouraging them to perform their maximum possible strength. The intraclass correlation coefficient (ICC) for the 1-RM bench press strength test was 0.966 (95\% confidence intervals $(\mathrm{CI})$ : lower $=0.914$, upper $=0.985$, coefficient of variation $(\mathrm{CV} \%)=12.8 \%)$.

Upper body rate of force development and isometric peak force: For the upper body RFD and isometric $\mathrm{PF}$, participants seated on a custom-made steel 
chair (hip angle of $100^{\circ}$ ) and placed their arms on a barbell (Figure 1), which was positioned with struts on the force plate (Applied Measurements Ltd Co., Reading, UK; WP800, A/D sampling frequency of $1 \mathrm{kHz}$ ). The barbell was positioned in parallel to the floor at the middle of the distance between the top of the shoulders and the lower point of the breastbone, allowing an angle of $90^{\circ}$ between the elbow and the armpits (Falvo et al., 2007; Hartmann et al., 2009). Real-time visual feedback of the force applied was provided for each effort via a computer monitor placed just above the force plate in front of the participants. During familiarization sessions, participants trained with 10 short-time attempts (1 s duration) to apply their force as fast as possible. Then, during the second visit, RFD measurement was performed. After a 5 min warm-up on a treadmill, light upper body stretching and 5-8 explosive push ups, participants performed two attempts with progressively increasing force and two fast attempts with approximately $80 \%$ of their maximum strength. Subsequently, three maximum efforts were performed of $3 \mathrm{~s}$ duration each, and $3 \mathrm{~min}$ rest intervals in between. Participants were instructed to apply their maximum force as fast as possible. Data from the force plate were recorded (Kyowa sensor interface PCD-320A) and analysed. Variables calculated from the force-time curve included the maximum isometric PF which was the greatest force generated from the force-time curve, and the RFD in specific time windows of $0-30,0-50,0-80,0-$ $100,0-150,0-200$, and $0-250 \mathrm{~ms}$, relative to the onset of contraction, which was set at $2.5 \%$ of the difference between baseline and maximum force (Zaras et al., 2021). The ICCs for the bench press RFD during repeated trials was: $\mathrm{ICC}_{30 \mathrm{~ms}}=0.838$ $(95 \%$ CI: lower $=0.610$, upper $=0.938, \mathrm{CV}=17.6 \%)$, ICC $_{50 \mathrm{~ms}}=0.845(95 \%$ CI: lower $=0.622$, upper $=$ $0.941, \mathrm{CV}=15.6 \%), \mathrm{ICC}_{80 \mathrm{~ms}}=0.815$ (95\% CI: lower $=0.567$, upper $=0.928, \mathrm{CV}=17.3 \%), \mathrm{ICC}_{100 \mathrm{~ms}}=$ $0.787(95 \%$ CI: lower $=0.514$, upper $=0.917, \mathrm{CV}=$ $17.9 \%), \mathrm{ICC}_{150 \mathrm{~ms}}=0.652(95 \% \mathrm{CI}$ : lower $=0.261$, upper $=0.859, \mathrm{CV}=17.5 \%), \mathrm{ICC}_{200 \mathrm{~ms}}=0.582(95 \%$ CI: lower $=0.143$, upper $=0.827, C V=18.4 \%)$ and $\mathrm{ICC}_{250 \mathrm{~ms}}=0.744(95 \%$ CI: upper $=0.427$, lower $=$ $0.899, \mathrm{CV}=18.0 \%)$. The ICC for maximum isometric PF was 0.869, (95\% CI: lower $=0.592$, upper $=0.961, \mathrm{CV}=16.4 \%$ ).

Triceps brachii ultrasonography: Panoramic B-mode ultrasound images were obtained with a $38-\mathrm{mm}$ linear probe from the long head of the triceps brachii using the "i-scape" software of the ultrasound device (10.0-MHz, Midray Z5, China). Initially, participants were placed at a standing position with their arms fully extended on the side of their body. The posterior surface of the acromion and the lateral epicondyle of the humerus were marked and the distance between them used as the total length of the upper arm (Reeves et al., 2004). The inner and outer regions of the arm were marked with a permanent pen at $60 \%$ (starting from the acromion). A self-adhesive paper was placed on the skin at this point, on the inner region of the arm, as a marker for the later analysis of the architecture (image shading). Then, participants laid supine with their arms rested on a laboratory bed at $90^{\circ}$ to their torso, fully extended at the elbow joint and supinated. A dashed line was drawn from the insertion of the triceps long head up to the medial epicondyle of the humerus. The transducer was placed along the dashed line and oriented in parallel to the muscle fascicles. The transducer's alignment was considered appropriate when several fascicles could be easily outlined without interruption across the image. Based on this orientation, a line $(\sim 10 \mathrm{~cm})$ was drawn on the left and the right of the point of $60 \%$ to identify and capture the largest continuous fascicle visualization (Noorkoiv et al., 2010; Stasinaki et al., 2018). To obtain the muscle image, a continuous single view was taken by moving the transducer along the marked line. However, due to individual differences, the transducer was sometimes aligned slightly diagonally to the longitudinal line of the muscle. Thus, after obtaining the images, two dots were marked on the skin, one on the left edge of marked line and one on the right edge. Coordinates of each edge of this dashed line were used to warrant the same measurement regions after the seven weeks of training. Images were analysed at $60 \%$ (as aforementioned) by image analysis software (Motic Images Plus, 2.0, Hong Kong) for muscle thickness, the fascicle angle, and fascicle length. Intersession reliability was determined by comparing the analysis of the images obtained by six participants (both arms) on two separate days when skin markings were completely removed. The ICC for the measurement of muscle thickness was 0.984 (95\% 
CI: lower $=0.951$, upper $=0.995, \mathrm{CV}=14.8 \%)$, for the fascicle angle was 0.858 (95\% CI: lower $=0.626$, upper $=0.952, \mathrm{CV}=17.0 \%$ ) and for fascicle length was $0.794(95 \%$ CI: lower $=0.483$, upper $=0.928$, $\mathrm{CV}=11.5 \%$ ).

\section{Statistical analysis}

For the determination of the sample size $(\mathrm{N}=16)$, an a priori power analysis $\left(\mathrm{G}^{*}\right.$ Power ver. 3.1) was performed according to previous description (Faul et al., 2007). Power analysis revealed that the actual power for the differences among groups was 0.967. All data are presented as mean $\pm \mathrm{SD}$ and were normally distributed according to the Kolmogorov-Smirnov test. A two-way repeated measures analysis of variance (ANOVA; 2 x 2 group $\times$ time), with Bonferroni post hoc correction, was used to evaluate differences for each variable between groups. Calculation of effect sizes $\left(\eta^{2}\right)$ was also performed, with the following interpretation of the effect size: small, $\eta^{2}=0.01$; medium, $\eta^{2}=0.06$; and large, $\eta^{2}=$ 0.14 (Cohen, 1988). Repeated measures analysis of covariance (ANCOVA) was used for the RFD performance test and triceps brachii muscle thickness because significant differences were found between the IRRG and TRG at pre-test measurements. An independent sample t-test was used to detect changes in the session RPE and training volume after inter-repetition rest and traditional training as well as to examine percentage differences between the IRRG and TRG. Reliability for all measurements was evaluated using a two way random effect intraclass correlation coefficient (ICC) with 95\% confident intervals (CI), as well as by calculating the coefficient of variation (CV\%). All data were analysed using SPSS 21 , and $p \leq 0.05$ was used as a 2-tailed level of significance.

\section{Results}

The mean session RPE was lower for the IRRG compared to the TRG (IRRG: $7.1 \pm 0.4$ vs. TRG: $8.1 \pm 0.4, p=0.000, \eta^{2}=0.630$ ) during the seven weeks of training. However, no significant difference was observed for the mean training volume between groups (IRRG: $1997.6 \pm 317.5 \mathrm{~kg}$ vs. TRG: $1872.2 \pm 163.4 \mathrm{~kg}, p=0.337, \eta^{2}=0.066$ ).

Changes in body mass, 1-RM strength, the upper body RFD and isometric PF are presented in Table 1. Body mass remained unaltered following the training period in both groups $(p=$
0.568, $\left.\eta^{2}=0.024\right)$. 1-RM bench press strength increased significantly in both groups, but interrepetition training induced a significantly higher percentage increase compared to traditional training (IRRG: $21.5 \pm 5.7 \%$ vs. TRG: $13.5 \pm 7.2 \%, p$ $\left.=0.027, \eta^{2}=0.294\right)$. ANCOVA revealed that the upper body RFD remained unchanged for the IRRG and TRG at all time frames of the force-time curve $(p>0.05)$. However, independent t-test analysis between percentage changes in the RFD revealed that inter-repetition training induced greater percentage increases during $0-80 \mathrm{~ms}$ and 0-100 ms compared to traditional training (RFD $80 \mathrm{~ms}:$ IRRG $=11.1 \pm 16.1 \%$ vs. TRG $=-4.2 \pm$ $6.6 \%, p=0.026, \eta^{2}=0.307$, RFD $100 \mathrm{~ms}:$ IRRG $=11.5 \pm$ $17.3 \%$ vs. TRG $=-3.2 \pm 7.2 \%, p=0.044, \eta^{2}=0.258$ ). Isometric PF remained unaltered for the TRG, but significantly increased for the IRRG (pre: $91.5 \pm$ $20.1 \mathrm{~kg}$, post: $100.2 \pm 18.3 \mathrm{~kg}, p=0.009, \eta^{2}=0.397$ ). However, no significant difference was observed between groups $\left(p=0.633, \eta^{2}=0.017\right)$.

Results for the triceps brachii muscle architecture are presented in Table 2. Both groups significantly increased triceps brachii muscle thickness with no difference between groups ( $p=$ $\left.0.107, \eta^{2}=0.175\right)$. Fascicle length and fascicle angle remained unaltered after seven weeks of training for both the IRRG and TRG.

\section{Discussion}

The aim of the current study was to compare inter-repetitions rest vs. traditional resistance training on upper body strength, the RFD and triceps brachii muscle architecture in physically active participants. Based on the acute and chronic benefits of inter-repetition rest training on upper body strength and velocity (Inglesias-Soler et al., 2014; Torrejón et al., 2019), it was hypothesised that inter-repetition rest training would induce greater increases in strength and the RFD compared to traditional resistance training. Indeed, the main finding of the present study was that inter-repetition rest training induced greater increases in 1-RM strength in the bench press compared to traditional training. In line with this result, only the IRRG isometric PF increased significantly. Although the upper body RFD remained unchanged for both training groups, comparisons of the percentage changes of the RFD between groups showed that inter-repetition rest training 
induced greater percentage increases in time frames of $0-80 \mathrm{~ms}$ and $0-100 \mathrm{~ms}$ compared to traditional training. These changes in performance were accompanied by increases in triceps brachii muscle thickness. Taken together, these results suggest that seven weeks of resistance training with $20 \mathrm{~s}$ inter-repetition rest intervals may contribute to higher increases in maximum strength and fast force production in active participants, compared to traditional resistance training.

\section{Table 1}

Changes in body mass, bench press 1-RM strength, the rate of force development and isometric peak force, after seven weeks of inter-repetition rest resistance training

or traditional resistance training.

\begin{tabular}{lcc|cc}
\hline & \multicolumn{2}{c|}{ Traditional Group } & \multicolumn{2}{c}{ Inter-Repetition Rest Group } \\
\hline Measurement & \multicolumn{1}{c|}{ Pre } & Post & Pre & Post \\
\hline Body mass & $79.5 \pm 12.8$ & $79.8 \pm 13.8$ & $75.7 \pm 8.4$ & $76.5 \pm 7.8$ \\
1-RM & $93.9 \pm 7.9$ & $106.5 \pm 10.18^{*}$ & $93.1 \pm 15.7$ & $113.1 \pm 19.7^{*}$ \\
RFD $_{30 \mathrm{~ms}}$ & $8790.2 \pm 3164.9$ & $8163.6 \pm 2317.0$ & $7025.1 \pm 1770.4$ & $7215.8 \pm 1505.2$ \\
RFD $_{50 \mathrm{~ms}}$ & $8700.2 \pm 2245.3$ & $8223.7 \pm 1777.4$ & $7383.7 \pm 1765.7$ & $7824.3 \pm 1370.5$ \\
RFD $00 \mathrm{~ms}$ & $7494.7 \pm 1080.4$ & $7173.9 \pm 1073.6$ & $6435.8 \pm 1582.4$ & $6973.3 \pm 1251.6$ \\
RFD $100 \mathrm{~ms}$ & $6672.6 \pm 705.8$ & $6471.2 \pm 895.3$ & $5732.3 \pm 1387.1$ & $6222.4 \pm 1091.4$ \\
RFD ${ }_{150 \mathrm{~ms}}$ & $5109.0 \pm 390.5$ & $4967.3 \pm 643.4$ & $4357.8 \pm 842.4$ & $4699.3 \pm 803.6$ \\
RFD $200 \mathrm{~ms}$ & $4006.5 \pm 336.9$ & $3865.3 \pm 515.3$ & $3408.2 \pm 596.3$ & $3639.1 \pm 685.3$ \\
RFD & $3211.5 \pm 443.7$ & $3214.3 \pm 377.7$ & $2848.2 \pm 472.1$ & $3049.6 \pm 579.8$ \\
IPF & $964.8 \pm 86.5$ & $1016.9 \pm 86.9$ & $897.1 \pm 197.1$ & $982.5 \pm 179.8^{*}$ \\
\hline
\end{tabular}

$1-R M=1$ Repetition Maximum in $\mathrm{kg}, \mathrm{RFD}=$ Rate of Force Development in $\mathrm{N} \cdot \mathrm{s}^{-1}, \mathrm{IPF}=$ Isometric Peak Force in $N$, body mass in kg. ${ }^{*} p<0.05$, difference between Pre and Post measurements.

Table 2

Changes in triceps brachii muscle architecture after seven weeks of inter-repetition rest resistance training or traditional resistance training.

\begin{tabular}{lcc|cc}
\hline \multicolumn{2}{c|}{ Traditional Group } & \multicolumn{2}{c}{ Inter-Repetition Rest Group } \\
\hline Measurement & Pre & Post & Pre & Post \\
\hline Thickness $(\mathrm{cm})$ & $1.99 \pm 0.24$ & $2.15 \pm 0.26^{*}$ & $1.76 \pm 0.32$ & $1.87 \pm 0.22^{*}$ \\
Fascicle length $(\mathrm{cm})$ & $9.7 \pm 1.7$ & $9.7 \pm 1.6$ & $9.7 \pm 1.0$ & $9.5 \pm 1.0$ \\
Fascicle Angle $\left({ }^{\circ}\right)$ & $15.3 \pm 2.8$ & $15.0 \pm 2.8$ & $14.2 \pm 4.4$ & $15.4 \pm 3.0$ \\
\hline
\end{tabular}

${ }^{*} p<0.05$, difference between Pre and Post measurements 


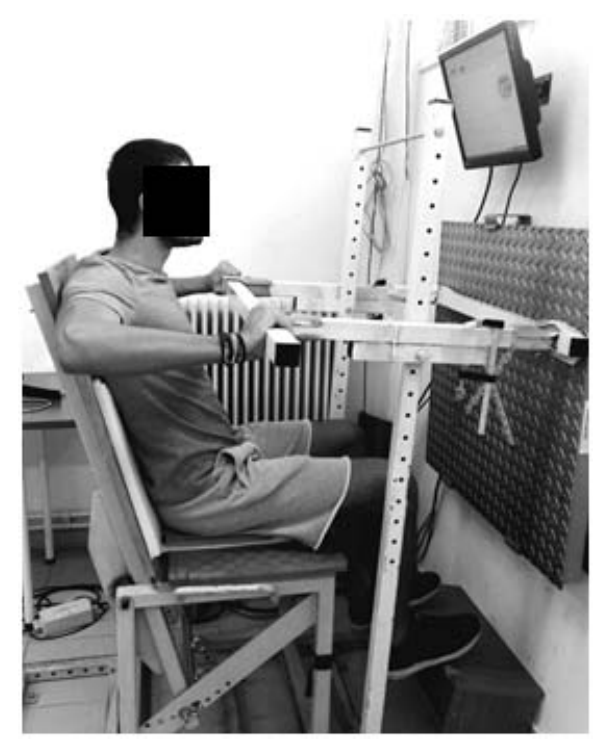

Figure 1

Seated upper body measurement for the evaluation of the rate of force development and isometric peak force.

Maximum strength in the bench press was significantly increased for both groups. Interrepetition rest training, however, induced higher percentage increases compared to traditional training, which is supported by a previous study (Oliver et al., 2013). Other studies did not observe any difference between inter-repetition rest or short interval configurations with traditional training intervention programs (Davies et al., 2019; Rial-Vázque et al., 2020), although some studies reported that traditional training may induce higher increases in upper body maximum strength than cluster training (Lawton et al., 2004; Rooney et al., 1994). However, most of the studies which reported a better outcome with traditional training included whole body training programs in male and female participants (Davies et al., 2019; Rial-Vázque et al., 2020), sometimes in athletic (Lawton et al., 2004) or military populations (Oliver et al., 2013). In the current study, a single, multijoint, commonly used exercise (bench press) was used in male participants of a similar initial strength level, to eliminate the above-mentioned methodological difficulties. In support of the higher effectiveness of inter-repetition rest training in increasing upper body strength, in the current study isometric PF was increased over-time only in the IRRG. This result further reinforces the finding that upper body maximum strength increased to a greater extent following inter-repetition rest training than traditional resistance training in active participants practicing a single upper body exercise.

The upper body RFD remained unchanged following both inter-repetition rest and traditional resistance training. This result may be explained by the fact that both groups performed each training repetition without the intention of maximum movement velocity during the concentric phase. Previous studies have showed that the intention of fast movement during the concentric phase may result in an increased RFD following resistance training (Blazevich et al., 2020). However, inter-repetition rest training induced higher percentage increases in the early RFD compared to traditional resistance training. This result may partially be explained by the potential advantage of the interrepetition rest training in maintaining a high velocity of movement in each repetition (Torrejón et al., 2019), which was not evaluated in the current study. Scarce data exist regarding changes in the upper body RFD after training. A previous 
study in physical education students revealed no significant change in the upper body maximum RFD after strength-power periodization training ( $7.06 \%$ increase) or daily-undulating periodization training (1.61\% increase) following 14 weeks of training (Hartmann et al., 2009). Nevertheless, it seems that adding $20 \mathrm{~s}$ inter-repetition rest intervals during bench press training may increase fast force production compared to traditional resistance training.

After seven weeks of training, significant increases were observed in triceps brachii muscle thickness for both the IRRG and TRG. The bench press is a multijoint exercise which includes the activation of pectoralis (pectoralis major, pectoralis minor), deltoid (medial and anterior deltoid) and arm muscles (long intern and extern portions of triceps brachii). Although triceps brachii contributes less to bench press strength compared to the chest muscles (Stastny et al., 2017), when fatigue occurs the activation of triceps brachii increases (Brennecke et al., 2009). In line with the present findings, previous resistance training studies of 12-24 week duration, reported significant increases in triceps brachii muscle thickness after training with a variety of resistance exercises including the bench press (Mata et al., 2012; Ogasawara et al., 2012). The current data suggest that inter-repetition rest resistance training enhances upper body hypertrophy similarly to traditional resistance training, in active participants, which may partly explain the increases in upper body strength and the RFD.

The findings of the present study should be interpreted with a certain degree of caution. A longer training-period intervention may potentially induce further changes in muscle strength, the RFD and triceps brachii muscle architecture, while a detraining period could potentially slow or stop this training adaptation. Additionally, neither electromyographic activity nor muscle biopsies were obtained in the current study, which would provide a better insight into the biological background accountable for the present results. Another limitation of the study was the relatively small sample size of participants in both training groups. Still, more research is needed to investigate the effect of inter-repetition rest training in well trained and/or athletic populations. Finally, training was performed without the intention of fast movement velocity during the concentric phase, while the actual velocity of repetitions was not measured for the IRRG and TRG, which might have provided a better insight into the RFD outcome. Although inter-repetition rest training maintains the high velocity of each repetition and may increase the RFD compared to traditional training, other studies should focus on the velocity of movement during inter-repetition rest resistance training.

\section{Conclusions}

The findings of the current study suggest that inter-repetition rest resistance training with single repetition configurations and $20 \mathrm{~s}$ interrepetition rest intervals induces greater percentage increases in upper body maximum strength and the RFD compared to traditional training. The bench press is a fundamental exercise for the development of upper body strength, and it is regularly used in most of the sports training routines. Resistance training with single repetitions in the bench press and $20 \mathrm{~s}$ inter-repetition rest intervals may increase maximum bench press strength which may result in enhanced upper body performance during an athletic-specific movement in several sports (i.e., shot put, discus throw, American football, etc), although more research is needed to reach certain conclusions. Additionally, the inter-repetition rest intervals increase fast force production to a greater extent than traditional resistance training and induce similar hypertrophy increases in triceps brachii muscle thickness. Consequently, coaches may use inter-repetition rest intervals especially during the pre-competitive period or during tapering for increasing rapid force production of the upper body. As a final point, set configurations with single repetitions induce lower to similar subjective feeling of exhaustion compared to traditional resistance training. Consequently, coaches may use inter-repetition rest configurations for upper body training without additional fatigue.

\section{Acknowledgements}

Authors express their gratitude to the participants of the study. 


\section{References}

Blazevich, A. J., Wilson, C. J., Alcaraz, P. E., \& Rubio-Arias, J. A. (2020). Effects of Resistance Training Movement Pattern and Velocity on Isometric Muscular Rate of Force Development: A Systematic Review with Meta-analysis and Meta-regression. Sports Medicine, 50, 943-963. https://doi.org/10.1007/s40279-019-01239-x

Brennecke, A., Guimarães, T. M., Leone, R., Cadarci, M., Mochizuki, L., Simão, R., Amadio, A. C., \& Serrão, J. (2009). Neuromuscular activity during bench press exercise performed with and without the pre exhaustion method. The Journal of Strength and Conditioning Research, 23(7), 1933-1940. https://doi: 10.1519/JSC.0b013e3181b73b8f

Borg, G. A. V. (1982). Psychophysical bases of perceived exertion. Medicine and Science in Sports and Exercise, 14(5), 377-381. https://doi.org/10.1249/00005768-198205000-00012

Cohen, J. (1988). Statistical Power Analysis for the Behavioral Sciences. Routledge Academic.

Davies, T. B., Halaki, M., Orr, R., Helms, E. R., \& Hackett, D.A. (2019). Changes in bench press velocity and power after 8 weeks of high-load cluster or traditional-set structures. The Journal of Strength and Conditioning Research, 34(10), 2734-2742. https://doi: 10.1519/JSC.0000000000003166

Day, M. L., McGuigan, M. R., Brice, G., \& Foster, C. (2004). Monitoring exercise intensity during resistance training using the session RPE scale. The Journal of Strength and Conditioning Research, 18(2), 353-358.

Falvo, M. J., Schilling, B. K., Bloomer, R. J., Smith, W. A., \& Creasy, A. C. (2007). Efficacy of prior eccentric exercise in attenuating impaired exercise performance after muscle injury in resistance trained men. The Journal of Strength and Conditioning Research, 21(4), 1053-1060. https://doi:10.1519/R-21406.1

Faul, F., Erdfelder, E., Lang, A. G., \& Buchner, A. (2007). G* power 3: A flexible statistical power analysis program for the social, behavioral, and biomedical sciences. Behavior Research Methods, 39, 175-191. https://doi.org/10.3758/BF03193146

Golas, A., Stronska, K., Krzysztofik, M., Stastny, P., \& Zajac, A. (2019). The influence of rest interval on total training load during 10 sets of the bench press exercise performed to concentric failure. Medicina Dello Sport, 72(2), 181-190. https://doi:10.23736/S0025-7826.19.03445-8

González-Badillo, J. J., Rodríguez-Rosell, D., Sánchez-Medina, L., Gorostiaga, E. M., \& Pareja-Blanco, F. (2014). Maximal intended velocity training induces greater gains in bench press performance than deliberately slower half-velocity training. European Journal of Sport Science, 14(8), 772-781. https://doi:10.1080/17461391.2014.905987

Haff, G. G., Hobbs, R. T., Haff, E. E., Sands, W. A., Pierce, K. C., \& Stone, M. H. (2008). Cluster training: A novel method for introducing training program variation. Strength and Conditioning Journal, 30(1), 6776. https:// doi:10.1519/SSC.0b013e31816383e1

Hardee, J. P., Triplett, N. T., Utter, A. C., Zwetsloot, K. A., \& Mcbride, J. M. (2012). Effect of inter-repetition rest on power output in the power clean. The Journal of Strength and Conditioning Research, 26(4), 883889. https://doi:10.1519/JSC.0b013e3182474370

Hartmann, H., Bob, A., Wirth, K., \& Schmidtbleicher, D. (2009). Effects of different periodization models on rate of force development and power ability of the upper extremity. The Journal of Strength and Conditioning Research, 23(7), 1921-1932. https://doi:10.1519/JSC.0b013e3181b73c69

Iglesias-Soler, E., Carballeira, E., Sanchez-Otero, T., Mayo, X., \& Fernandez-Del-Olmo, M. (2014). Performance of maximum number of repetitions with cluster set configuration. International Journal of Sports Physiology and Performance, 9(4), 637-642. https://doi.org/10.1123/ijspp.2013-0246

Krzysztofik, M., Jarosz, J., Matykiewicz, P., Zajac, A., \& Golas, A. (2021). A comparison of muscle activity of the dominant and non-dominant side of the body during low versus high loaded bench press exercise performed to muscular failure. Journal of Electromyography and Kinesiology, 56, 102513. https://doi.org/10.1016/j.jelekin.2020.102513

Komi, P. V. (2003). Strength and Power in Sport (2 ${ }^{\text {nd }}$ Ed.). Blackwell Science. http://www.blackwellpublishing.com

Lawton, T., Cronin, J., Drinkwater, E., Lindsell, R., \& Pyne, D. (2004). The effect of continuous repetition training and intra-set rest training on bench press and power. Journal of Sports Medicine and Physical Fitness, 44(4), 361-367.

Matta, T., Simão, R., de Salles, B. F., Spineti, J., \& Oliveira, L.F. (2011). Strength training's chronic effects on

(c) Editorial Committee of Journal of Human Kinetics 
muscle architecture parameters of different arm sites. The Journal of Strength and Conditioning Research, 25(6), 1711-1717. https://doi:10.1519/JSC.0b013e3181dba162

Miller, T. (2012). NSCA's Guide to test assessments. Human Kinetics. www.HumanKinetics.com

Mora-Custodio, R., Rodríguez-Rosell, D., Yáñez-García, J. M., Sánchez-Moreno, M., Pareja-Blanco, F., \& González-Badillo, J. J. (2018). Effect of different inter-repetition rest intervals across four load intensities on velocity loss and blood lactate concentration during full squat exercise. Journal of Sports Sciences, 36(14), 2856-2864. https://doi.org/10.1080/02640414.2018.1480052

Nicholson, G., Ispoglou, T., \& Bissas, A. (2016). The impact of repetition mechanics on the adaptations resulting from strength-hypertrophy and cluster-type resistance training. European Journal of Applied Physiology, 116, 1875-1888. https://doi.org/10.1007/s00421-016-3439-2

Noorkoiv, M., Stavnsbo, A., Aagaard, P., \& Blazevich, A. (2010). In vivo assessment of muscle fascicle length by extended field-of-view ultrasonography. Journal of Applied Physiology, 109(6), 1974-1979. https://doi.org/10.1152/japplphysiol.00657.2010

Ogasawara, R., Thiebaud, R. S., Loenneke, J. P., Loftin, M., \& Abe, T. (2012). Time course for arm and chest muscle thickness changes following bench press training. Interventional Medicine and Applied Science, 4(4), 217-220. https://doi.org/10.1556/imas.4.2012.4.7

Oliver, J. M., Jagim, A. R., Sanchez, A. C., Mardock, M. A., Kelly, K. A., Meredith, H. J., Smith, L. G., Greenwood, M., Parker, L. J., Riechman, E. S., Fluckey, D. J., Crouse, F. S., \& Kreider, B. R. (2013). Greater gains in strength and power with intraset rest intervals in hypertrophic training. The Journal of Strength and Conditioning Research, 27(11), 3116-3131. https://doi:10.1519/JSC.0b013e3182891672

Oliver, J. M., Kreutzer, A., Jenke, S., Phillips, M. D., Mitchell, J. B., \& Jones, M.T. (2015). Acute response to cluster sets in trained and untrained men. European Journal of Applied Physiology, 115, 2383-2393. https://doi.org/10.1007/s00421-015-3216-7

Reeves, N., Maganaris, C., \& Narici, M. (2004). Ultrasonographic assessment of human skeletal muscle size. European Journal of Applied Physiology, 91, 116-118. https://doi.org/10.1007/s00421-003-0961-9

Rial-Vázquez, J., Mayo, X., Tufano, J. J., Fariñas, J., Rúa-Alonso, M., \& Iglesias-Soler, E. (2020). Cluster vs. traditional training programmes: changes in the force-velocity relationship. Sports Biomechanics. https://doi.org/10.1080/14763141.2020.1718197

Rooney, K. J., Herbert, R. D., \& Balnave, R. J. (1994). Fatigue contributes to the strength training stimulus. Medicine and Science in Sports and Exercise, 26(9), 1160-1164.

Stasinaki, A-N., Zaras, N., Methenitis, S., Tsitkanou, S., Krase, A., Kavvoura, A., \& Terzis, G. (2018). Triceps Brachii Muscle Strength and Architectural Adaptations with Resistance Training Exercises at Short or Long Fascicle Length. Journal of Functional Morphology and Kinesiology, 3(2), 28. https://doi:10.3390/jfmk3020028

Stastny, P., Gołaś, A., Blazek, D., Maszczyk, A., Wilk, M., Pietraszewski, P., Petr, M., Uhlir, P., \& Zając, A. (2017). A systematic review of surface electromyography analyses of the bench press movement task. PLoS ONE, 12(2). doi: 10.1371/journal.pone.0171632

Sweet, T. W., Foster, C., McGuigan, M. R., \& Brice, G. (2004). Quantitation of resistance training using the session rating of perceived exertion method. The Journal of Strength and Conditioning Research, 18(4), 796-802. https://doi:10.1519/14153.1. PMID: 15574104

Torrejón, A., Janicijevic, D., Haff, G. G., \& García-Ramos, A. (2019). Acute effects of different set configurations during a strength-oriented resistance training session on barbell velocity and the forcevelocity relationship in resistance-trained males and females. European Journal of Applied Physiology, 119, 1409-1417. https://doi.org/10.1007/s00421-019-04131-8

Zaras, N., Stasinaki, A-N., Spiliopoulou, P., Arnaoutis, G., Hantjicharalambous, M., \& Terzis, G. (2021). Rate of force development, muscle architecture and performance in elite weightlifters. International Journal of Sports Physiology and Performance, 16(2), 216-223. https://doi.org/10.1123/ijspp.2019-0974

\section{Corresponding author:}

Nikolaos Zaras (MSc, PhD),

Human Performance Laboratory, Department of Life \& Health Sciences, University of Nicosia, 46 Makedonitissas Ave., CY-2417, P.O.Box 24005, 1700 NICOSIA, CYPRUS,

Tel.: 00357 22842318; Fax.: 0035722842399; E-mail: zaras.n@unic.ac.cy 